\title{
Von der Therapiestudie zur Qualitätssicherung in der Pädiatrischen Onkologie
}

\author{
U. Göbel, R. Fischer und G. Henze
}

Trotz langjähriger und intensiver Bemühungen ist die Sterblichkeit an bösartigen Erkrankungen im Erwachsenenalter noch ohne sicher erkennbaren Rückgang (1). Allerdings ist auch keine weitere $\mathrm{Zu}$ nahme der Gesamtmortalität zu verzeichnen, wie dies z. B. aufgrund der weiterhin kontinuierlich steigenden Lebenserwartung, der unverändert riskanten Lebens- und Genußgewohnheiten oder einseitiger Ernährung zu erwarten ist. Vor dieser stagnierenden Bilanz des Erwachsenenalters sind die inzwischen erreichten guten Behandlungsergebnisse bei bösartigen Erkrankungen im Kindesalter zu sehen, die in Deutschland und anderen westlichen Industrienationen durch Langzeitremissionen von durchschnittlich $70 \%$ gekennzeichnet sind (8).

Da ähnlich gute Behandlungsergebnisse im Erwachsenenalter nur für den Morbus Hodgkin und die malignen Hodentumoren erzielt werden können, sprechen die bei Kindern allgemein dokumentierten Erfolge für die eminente Bedeutung der Chemosensibilität der bei Kindern vorkommenden bösartigen Erkrankungen (Abb. 1), die sich erheblich von den Diagnosen des Erwachsenenalters unterscheiden. Die als Vergleichsmaßstab gewählten Zweijahresremissionsraten geben noch keine Auskunft über den definitiven Heilungserfolg, lassen aber eindrucksvoll die in den vergangenen Jahrzehnten erzielten Fortschritte erkennbar werden und ermöglichen gleichzeitig eine Zuordnung zu wichtigen therapeutischen Innovationen (5): Sind die Verbesserungen der 30er und 40er Jahre wesentlich durch die Fortschritte der Chirurgie und Anästhesie bedingt, so sind die der 50er Jahre auf den ersten Einsatz von Zytostatika zurückzuführen. In den $60 \mathrm{er}$ Jahren folgten die ersten programmatischen Therapieprotokolle, in denen die zeitliche Abfolge von Operation, Bestrahlung und Chemotherapie - meist in Form einer Sequenz von 2 oder 3 Zytostatika - festgelegt wurde. Die zeitgleiche Verabfolgung von Zytostatikakombinationen und Steigerung der einzelnen Zytostatikagabe bis in den Hochdosisbereich sowie die präoperative bzw. neoadjuvante Chemotherapie waren die Innovationen der $70 \mathrm{er}$ und $80 \mathrm{er}$ Jahre, während die $90 \mathrm{er}$ Jahre durch die zunehmende Zahl autologer und allogen-verwandter bzw. allogenunverwandter hämatopoetischer Stammzelltransplantationen gekennzeichnet sind.

Die in vielen kleinen Einzelschritten erreichten therapeutischen Erfolge wären ohne die parallel erarbeiteten Fortschritte in der Diagnostik und die Entwicklung effektiver Supportivmaßnahmen undenkbar gewesen. Deshalb haben die Begriffe der multimodalen Diagnostik und Therapie sowie die Hochdosistherapie schon frühzeitig Eingang in den Sprachgebrauch und den klinischen Alltag der Pädiatrischen Onkologie gefunden (6).

Da die Therapiestudien in den ersten Jahrzehnten ihrer Anwendung neben dem individuellen Heilbemühen vor allem auch dem Erkenntnisgewinn durch das Studium der Erkrankungen unter einheitlichen Therapiebedingungen dienten. waren sie das wesentliche Element der klinischen Forschung innerhalb der Pädiatrischen Onkologie. Die zunehmend bessere Kenntnis des biologischen Verhaltens einer bestimmten malignen Erkrankung unter standardisierten Therapiebedingungen und die therapicbegleitende Evaluierung prätherapeutisch definierter Risikofaktoren ermöglichten konsekutiv eine zunehmende Stratifizicrung der Behandlung. Dies bedeutete eine schrittweise Zurücknahme bestimmter Therapiemaßnahmen bej Kindern mit relativ günstiger Prognose und meist eine Eskalation der Therapieintensität bei Kindern mit besonders ungünstigen Krankheitsvorzeichen. Dieser innerhalb von drei Jahrzehnten eingetretene Wandel fühte dazu, daß heutzutage an Stel-

Klin. Pädiatr. 209 (1997) 145-146

(1) 1997 F. Enke Verlag Stuttgart le von Therapiestudien von Therapieoptimierungsstudien gesprochen wird (2). Inhaltlich handelt es sich um sorgfältig erstellte Diagnostik- und Therapiepläne, die in Deutschland bei Kindern und Jugendlichen mit bösartigen Erkrankungen flächendeckend eingesetzt und zentral ausgewertet werden. Sie erfüllen damit in hervorragender Weise die Kriterien einer freiwilligen Qualitätskontrolle (4), die durch eine

- nahezu vollständige Erfassung aller bösartigen Neuerkrankungen bei Kindern und Jugendlichen im Kinderkrebsregister Mainz

- zentrale Referenzdiagnostik unter Anwendung einheitlicher histologischer, histochemischer oder immunphänotypischer bzw. zytogenetischer oder molekularbiologischer Untersuchungstechniken und

- standardisierte Behandlung unter Berücksichtigung individueller Besonderheiten gekennzeichnet ist.

Insofern ist es nicht verwunderlich, daß die im Auftrag des Bundesministers für Gesundheit in Vorbereitung befindlichen Leitlinien zur Diagnostik und Therapie von Erkrankungen im Bereich der pädiatrischen Onkologie den erreichten hohen Standard dieser Diagnostik- und Behandlungsprotokolle wiedergeben (3). Trotz dieser Leitlinien werden aber auch weiterhin nur durch die Teilnahme des einzelnen Patienten an einem festen Behandlungsprotokoll unter Nutzung der zentralen Qualitätskontrolle und Inanspruchnahme der zentralen Konsiliartätigkeit die prinzipiell vorhandenen Behandlungschancen in der bestmöglichen Weise realisiert, wie die Behandlungsergebnisse in anderen Industrienationen gezeigt haben (9). Hierbei besteht eine stete Interdependenz zwischen diagnostizierender und behandelnder Einrichtung, die auch die Aufgabe der individuellen Dokumentation zu erfüllen hat. dem Kinderkrebsregister im Institut für Medizinische Statistik und Dokumentation der Universität Mainz und der für die jeweilige Erkrankung zuständigen Studienleitung (Abb. 2)

In den Anfangsjahren hatte das damalige Bundesministerium für Forschung und Technologie die pädiatrischen Therapiestudien als Modellmaßnahmen in großzügiger Weise und mit unverkennbarem Erfolg gefördert. Nach der termingerechten Beendigung der mo-

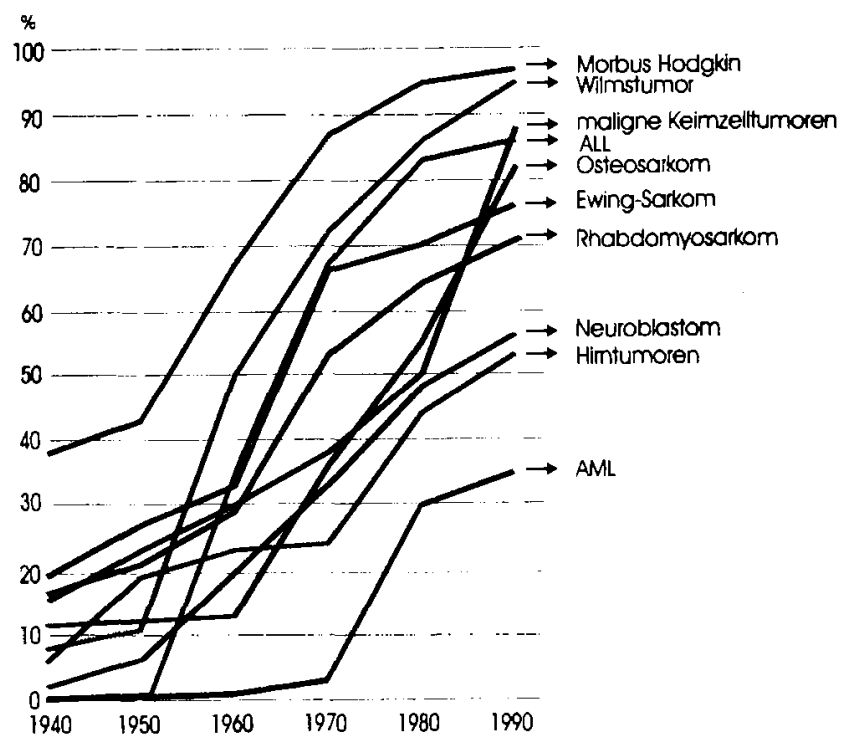

Abb. 1 Zwei-Jahres-Überlebensraten von Kindern und Jugendlichen mit bösartigen Erkrankungen 
Tumordokumentation bei Kindern

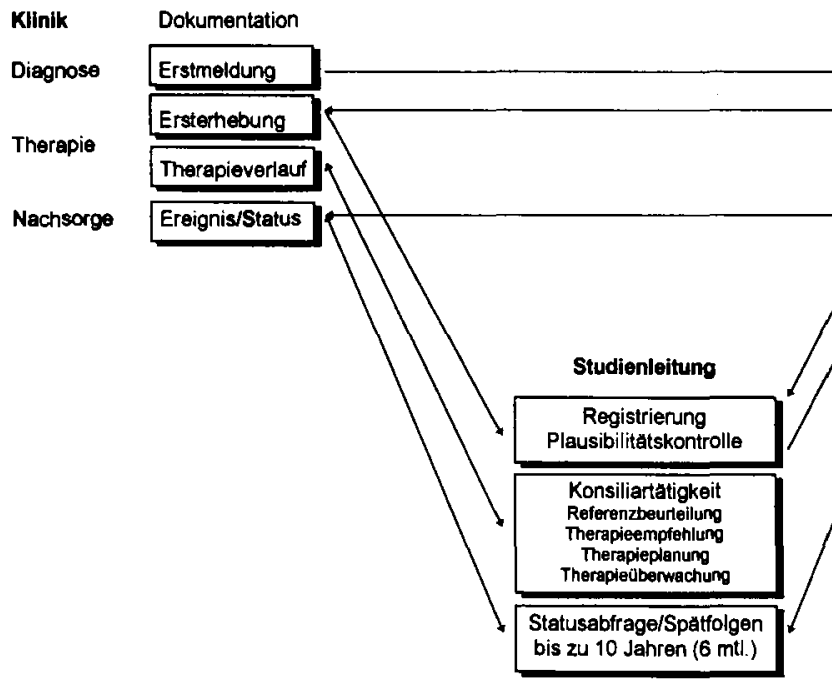

dellhaften Förderung durch die Bundesregierung der Bundesrepublik Deutschland hat sich die Deutsche Krebshilfe aus innerer Überzeugung zur Weiterförderung bereit erklärt, da ihr die Pädiatrische Onkologie ein besonderes Anliegen ist und die erzielten Fortschritte zum Wohle der Kinder nicht infrage gestellt werden dürfen $(7,10)$.

Mit Erreichen des jetzigen hohen Behandlungsstandards ist eine dauerhafte Lösung im Sinne der Qualitätssicherstellung zu schaffen. da dies nicht die zeitlich unbegrenzte Aufgabe einer Bürgerinitiative, wie sie die Deutsche Krebshilfe darstellt, sein kann. Dies bedeutet nicht, daß sich die Deutsche Krebshilfe aus der Förderung der Pädiatrischen Onkologie zurückziehen möchte, sondern verdeutlicht vielmehr die Absicht, nach Etablierung einer alternativen Finanzierung die freiwerdenden Spendenmittel vermehrt auch für andere, innovative Forschungsansätze zu verwenden: Gemeinsames Ziel ist es, auch dem Drittel krebskranker Kinder zu helfen, die mit den bisherigen Therapiemaßnahmen nicht überleben können. Große Hoffnungen werden hierbei in die kliniknahe Grundlagenforschung gesetzt, um über bessere Kenntnisse der Ätiopathogenese der bösartigen Erkrankungen im Kindesalter neue Therapieoptionen zu erarbeiten.

\section{U. Göbel}

Mitglied des Med. Beirates der Deutschen Krebshilfe und des Beirates der Gesellschaft für Pädiatrische Onkologie und Hämatologie

R. Fischer

Vorsitzender des Med. Beirates der Deutschen Krebshilfe

G. Henze

Vorsitzender der Gesellschaft für Pädiatrische Onkologie und Hämatologie

\section{Literatur}

${ }^{1}$ Bailar, J. C., H. L. Gornik: Cancer Undefeated. New Engl. J. Med. 336 (1977) 1569

2 Creutzig, U., K. Winkler: Empfehlungen für Studien zur Optimierung von Therapieschemata. Klin. Pädiatr. 206 (1994) 191-193

"Creuzzig, U.. K. Winkler, G. Henze: Leitlinien für die Diagnostik und Therapie in der Pädiatrischen Onkologie und Hämatologie. Klin. Pädiatr. 208 (1996) 139-140

${ }^{4}$ Enghofer, E., K. Winkler (Hrsg.): Qualitätssicherung in der Onkologie Grundlagen und Definition. W. Zuckschwerdt-Verlag (1995)

s Göbel, U.: Prinzipien hei der Bchandlung von Neoplasien im Kindes- und Jugendalter. Monatsschr. Kinderheilkd. $1.3,(19,7)+6,3-+11$
Abb. 2 Interaktion zwischen behandelnder Klinik, Kinderkrebsregister (Institut für Medizinische Statistik und Dokumentation der Universität Mainz) und Leitung der Therapieoptimierungsstudie

- Göbel, U., H. Jürgens, D. Schwamborn, V. Wahn: Hochdosierte Chemotherapie: Grundlagen. Indikationen. Komplikationen. Klin. Pädiatr. 200 (1988) $1-6$

7 Jonat, W., U. Göbel: Fortschritte durch Therapiestudien. Münch. med. Wschr. 136 (1994) 601-604

* Kaletsch, U., P. Kaatsch, J. Michaelis: Jahresbericht 1995 des Deutschen Kinderkrebsregisters. Institut für Medizinische Statistik und Dokumentation, Universität Mainz (1996)

" Murphy, S. B.: The National Impact of Clinical Cooperative Group Trials for Pediatric Cancer. Med. Ped. Oncol. 24 (1995) 279-280

11 Winkler, K.: 20 Jahre Deutsche Krebshilfe e. V. Klin. Pädiatr. 206 (1995) $189-190$

\section{Ergebnisse der Gesellschaft fïr Pädiatrische Onkologie und Hämatologie (GPOH)}

1977 veröffentlichte die Zeitschrift "Klinische Pädiatrie" erstmals die Verhandlungen der Deutschen Arbeitsgemeinschaft für Leukämieforschung und Behandlung im Kindesalter e.V. (DAL) in einem regulären Heft als Ergebnisse der Pädiatrischen Onkologie 1. Dieses großzügige Angebot ist zu einer festen Einrichtung geworden, obwohl sich zwischenzeitlich vielfältige Entwicklungen mit der Gründung der Gesellschaft für Onkologie (GPO) im Jahre 1974 und der 1991 erfolgten Fusion von DAL und GPO zur Gesellschaft für Pädiatrische Onkologie und Hämatologie unter Hinzunahme eines neuen Forschungsschwerpunktes ergeben haben.

Die Gesellschaft für Kinderheilkunde bietet in der Monatsschrift für Kinderheilkunde die Möglichkeit an, Abstracts von wissenschaftlichen Tagungen zu veröffentlichen, damit die Entwicklungen innerhalb der verschiedenen pädiatrischen Arbeitsgemeinschaften bzw. Gesellschaften allen ihren Mitgliedern zugänglich sind. Von diesem Angebot macht die GPOH seit 1994 Gebrauch, indem sie regelmäßig und termingerecht seit 1995 die Abstracts zu den Halbjahrestagungen in der Monatsschrift für Kinderheilkunde in deutscher Sprache veröffentlicht. Diese Veröffentlichung in deutscher Sprache trägt dem Sachverhalt Rechnung, daß es sich um eine deutschsprachige Tagung handelt.

Darüberhinaus gewinnen aber die Aktivitäten der GPOH im Ausland zunehmend an Interesse. Dies äußert sich $u$. a. auch darin, daB die Abstracts der Jahrestagung der Kind-Philipp-Stiftung für Leukämie-Forschung seit 1990 in dem Jahresheft "Ergebnisse der Pädiatrischen Onkologie" in englischer Sprache publiziert werden. Hierdurch werden die forscherischen Aktivitäten auch international dargestellt und verfügbar gemacht.

In konsequenter Weiterverfolgung dieses Gedankens werden in diesem Heft alle Beiträge in englischer Sprache publiziert, ohne dal3 hierdurch jedoch die lntention dieser ständigen Einricht ung verändert werden soll: Die Berichte der Gesellschaft für Pädiatrische Onkologie und Hämatologie sollen allen Mitgliedern. darüberhinaus aber auch im zusammenwachsenden Europa allen Interessierten, in ausführlicher Weise zugänglich gemacht werden. Dies erleichtert nicht nur die Darstellung unserer geleisteten Arbeit, sondern auch rukünftige grenzüberschreitende Kooperationen 